IOS Press

\title{
Online Personalised Training in Memory Strategies for Everyday (OPTIMiSE) Program for Older Adults with Cognitive Concerns: Pilot Study Protocol
}

\author{
Kerryn E. Pike ${ }^{\mathrm{a}, *}$, Carl I. Moller ${ }^{\mathrm{a}}$, Christina Bryant ${ }^{\mathrm{b}}$, Maree Farrow ${ }^{\mathrm{c}}$, \\ Duy P. Dao a and Kathryn A. Ellis ${ }^{\mathrm{b}, \mathrm{d}, \mathrm{e}}$ \\ ${ }^{a}$ School of Psychology and Public Health, La Trobe University, Melbourne, Australia \\ ${ }^{\mathrm{b}}$ Melbourne School of Psychological Sciences, The University of Melbourne, Melbourne, Victoria, Australia \\ ${ }^{\mathrm{c}}$ Wicking Dementia Research and Education Centre, University of Tasmania, Hobart, Tasmania, Australia \\ ${ }^{\mathrm{d}}$ Academic Unit for Psychiatry of Old Age, Department of Psychiatry, The University of Melbourne, \\ Melbourne, Victoria, Australia \\ ${ }^{\mathrm{e}}$ Florey Institute for Neuroscience and Mental Health, The University of Melbourne, Melbourne, \\ Victoria, Australia
}

Accepted 28 January 2021

Pre-press 19 February 2021

\begin{abstract}
Memory interventions for older adults with cognitive concerns result in improved memory performance and maintenance of cognitive health. These programs are typically delivered face-to-face, which is resource intensive and creates access barriers, particularly for those with reduced mobility, limited transportation, and living in rural or remote areas. The COVID-19 pandemic has created an additional access barrier, given the increased risk this disease poses to older adults. Internet-based interventions seek to overcome these barriers. This paper describes the protocol of a pilot study that aims to evaluate the feasibility, acceptability, and efficacy of one such internet-based intervention: the Online Personalised Training in Memory Strategies for Everyday (OPTIMiSE) program. OPTIMiSE focuses on improving knowledge regarding memory and providing training in effective memory strategies for everyday life. The pilot study described in this protocol will be a single-arm pre-post study of 8 weeks duration, with a single maintenance session 3 months post-intervention. Participants will be Australian adults aged $\geq 60$ years reporting cognitive changes compared with 10 years ago. Primary outcome measures will address feasibility, acceptability, and efficacy. Secondary outcome measures assessing sense of community and self-efficacy will be administered at the 8-week and 3-month timepoints. Data collection will conclude mid-2021, and results will be presented in a subsequent publication. Translation of memory interventions to internet-based delivery has the potential to remove many access barriers for older adults; however, the acceptability and feasibility of this modality needs investigation. OPTIMiSE is the initial step in what could be an important program enabling access to an evidence-based memory intervention for older adults worldwide.
\end{abstract}

Trial registration: Australian New Zealand Clinical Trials Registry (ANZCTR), ACTRN12620000979954

Keywords: Aged, aging, cognition, learning, internet-based intervention, memory, self efficacy, social support

\footnotetext{
${ }^{*}$ Correspondence to: Dr. Kerryn Pike, School of Psychology \& Public Health, La Trobe University, Melbourne, Victoria, 3086, Australia. Tel.: +61 39479 1381; Fax : +61 39479 1956;
}

E-mail: k.pike@latrobe.edu.au.; ORCID: http://orcid.org/00000002-0474-1215 


\section{INTRODUCTION}

Concerns about declining cognition, particularly memory, are common among older adults with community prevalence ranging from $33-95 \%$, depending on the measure used $[1,2]$. These cognitive concerns can occur with objective cognitive impairment (mild cognitive impairment; MCI) or without (subjective cognitive decline; SCD). Both MCI and SCD are associated with increased risk of developing dementia [3,4], as well as being linked to heightened distress [5] and reduced quality of life [6, 7]. Many studies have examined cognitive interventions-particularly for memory-for older adults with MCI and SCD, with meta-analyses demonstrating modest improvements in cognitive test performance [8,9], as well as positive changes in perceived memory ability, selfefficacy, strategy use, memory-related affect, psychological well-being, quality of life, mood, and activities of daily living [10-12].

Cognitive interventions have taken two broad approaches: a) a restorative approach, involving repeated practice on a discrete cognitive task in order to improve performance in that domain (cognitive or "brain" training), and b) a compensatory approach, involving training in specific compensatory cognitive strategies. One limitation of cognitive training interventions is the lack of transfer, particularly when considering performance in daily life [13]. Suggestions to redress the issue of poor transfer of memory training to everyday life include targeting training to everyday memory tasks that older adults have dificulties with and are motivated to improve, i.e., a personalized approach using ecologically relevant tasks [14]. Another limitation of many existing intervention studies is the focus on cognitive test performance as the primary outcome, despite participants valuing improved self-efficacy over test performance [15]. Three aspects of memory interventions are considered important for improving self-efficacy and everyday memory performance. These are psychoeducation regarding age-related changes in memory, training in multiple memory strategies, and opportunity to share experiences of memory complaints among peers [16]. Furthermore, practice in daily life, and engagement in such practice over an extended period (an average of 2 months) has been shown to be important in facilitating the creation of new habits or behavior change [17].

One example of an effective cognitive intervention targeted to older adults with SCD and MCI is the La Trobe and Caulfield Hospital (LaTCH) memory management group program [18]. This program has a compensatory approach, aiming to improve memory in daily life, rather than memory impairment as measured by test performance. This manualized group program runs for 2 hours per week over 6 weeks. The program's goal directed problem-solving approach fits within a cognitive behavioral therapy framework. It provides psychoeducation regarding memory, as well as teaching evidence-based strategies and their practical application in everyday life, through in-session demonstration, practice, and take-home exercises. A problem-solving approach is espoused where participants are guided to identify a target memory issue, consider why the issue is occurring (after psychoeducation regarding memory), identify the best strategy for their issue from the range of strategies discussed, and then tweak the chosen strategy as needed. The program's efficacy has been demonstrated through two randomized trials $[18,19]$, a five-year follow-up study [20], and translation to practice with a community organization [21]. Program benefits include improved 1) knowledge of memory strategies; 2) use of memory strategies; 3 ) self-reported memory ability; and 4) memory contentment and self-efficacy. Furthermore, participants report normalization of their memory concerns, realization that others experience similar difficulties, acceptance of their memory ability, and feelings of enhanced coping and selfefficacy, which highlight the importance of the group aspect of the intervention [20]. Similar outcomes have been demonstrated by other memory group programs (for example, [22]; see also [12] for further examples).

Despite these positive outcomes, challenges arise when trying to implement these programs in realworld settings. These challenges include having the resources, or establishing sustainable funding, for staffing and facilitator training, as well as managing administrative demands [21]. Facilitator training can be an ongoing issue due to staff turnover [21]. Furthermore, face-to-face memory interventions limit access to older adults who can attend a specific location at set dates and times [16]. Given the association between age and chronic health conditions, older adults may be limited in their ability to attend clinics for face-to-face interventions, particularly across multiple sessions. This has been intensified by the COVID-19 pandemic, and heightened risk of mortality for older adults [23], meaning this population is likely to avoid any non-urgent, face-to-face contact, particularly in group situations. Accessibility of face-to-face groups is also limited for older adults 
with reduced mobility, limited transport options, or residing in rural and remote regions.

One possible solution to both service delivery and access issues is an online program addressing memory strategy training [16], which is the focus of this pilot study of the Online Personalised Training in Memory Strategies for Everyday (OPTIMiSE) program. OPTIMiSE will be developed with similar goals to the LaTCH memory management group program, aiming to 1) improve knowledge regarding memory, and 2) provide effective memory strategies for everyday life. OPTIMiSE will also have a similar problem-solving approach to LaTCH. Program content will be broadly like that covered in LaTCH, with a focus on everyday examples of memory difficulties (such as remembering names, losing items, prospective memory), along with a range of practical evidence-based strategies appropriate for each issue. Additionally, OPTIMiSE will aim to develop a sense of community among participants, through sharing their own memory concerns, experiences, and strategies in moderated online discussion boards. In contrast to LaTCH, where all participants are provided with the same content, the OPTIMiSE program will be personalized, with participants being able to tailor the program content to suit their self-perceived memory deficits.

Increasingly, the internet is used by consumers to access health information, including online psychological interventions, which have been shown to be safe, feasible, and efficacious [16]. Older adult internet use has been rising every year, with $83 \%$ of Australians aged 55-64, and 55\% of Australians over 65 years using the internet [24]. The COVID-19 pandemic, and the resultant social distancing and isolation measures designed to limit the spread of the virus, highlight the potentially important role of telehealth or online models of health service provision. Eliminating the need for face-toface contact for health service delivery is especially important for older adults [23]. The current global pandemic presents many significant challenges, but also provides a valuable opportunity to assess the implementation of an online memory strategy program for older adults. Notably, a recent Australian survey suggested that of eight proposed online health interventions, the one of most interest to older adults was memory strategy training, with $82 \%$ of the sample indicating they would utilize this if available [25]. Online memory interventions for older adults appear to be feasible [16], although most attempts to provide memory interventions online to date have been restricted to restorative or cognitive training interventions. The OPTIMiSE program has been designed to address issues of sustainability of service delivery and enable wider access to a compensatory program of evidence-based memory strategies for older adults, with greater ecological validity than restorative "brain training" programs.

This paper describes the protocol of the OPTIMiSE pilot study, the primary aim of which will be to evaluate the feasibility (recruitment, attrition, data collection), acceptability, and efficacy of the OPTIMiSE intervention. We want to determine whether it will be feasible to run OPTIMiSE for older adults with cognitive concerns on a larger scale. We also want to explore whether OPTIMiSE will achieve similar outcomes to face-to-face programs such as the LaTCH memory management group, i.e., moderate to large effect size changes in memory strategy knowledge and use, memory ability, and satisfaction with memory. Consistent with other memory interventions [18], we expect improvement in participants' knowledge regarding memory and ageing, strategy knowledge, strategy use, memory ability, satisfaction with their memory, and improvement on individual memory goals. Furthermore, the secondary aim of this pilot study will be to explore the ability of OPTIMiSE to create a sense of community among participants, whether the sense of community engendered through OPTIMiSE is sufficient to normalize participants' memory concerns, and if the program is able to foster and enhance feelings of self-efficacy and control with respect to memory ability [20]. It is anticipated that outcome data from the pilot study will be reported in a subsequent publication.

\section{METHODS}

\section{Study design}

The OPTIMiSE pilot is a single-arm pre-post study of 8 weeks duration, with a single maintenance session 3 months post-intervention. The study has been registered with the Australian New Zealand Clinical Trials Registry (ACTRN12620000979954) and has approval from relevant institutional Human Research Ethics Committees. As the pilot study employs a single-arm pre-post design, there is no separate control group. A pre-post design is a commonly used, pragmatic choice in health service evaluation, particularly when gathering preliminary evidence around intervention efficacy [26], which is a primary aim of the pilot study. While not providing the same level of 
scientific rigor as an experimental study design such as a randomized controlled trial, the chosen design offers many advantages with respect to the simplicity and cost effectiveness of participants serving as their own controls. This pilot was designed to maximize the use of limited resources, thus a pre-post design strikes an acceptable balance of scientific rigor and practicality. One of the core aims of this pilot is to evaluate the feasibility of upscaling OPTIMiSE for delivery to a larger cohort in the future. With this in mind, outcomes of the pilot study will be reported in accordance with both the STROBE (Strengthening the Reporting of Observational Studies in Epidemiology) guidelines [27] and the CONSORT (Consolidated Standards of Reporting Trials) extension for pilot and feasibility trials [28], with adaptation of items where necessary to reflect the non-randomized design of the OPTIMiSE intervention and to ensure that all reporting items relate to issues of feasibility [29].

\section{Participants}

Participants will be recruited across all states and territories of Australia through emails directed to community groups of older adults in Australia. Avenues of recruitment will include U3A (University of the Third Age) communities, Probus Clubs, and people who had previously expressed interest in participating in the face-to-face LaTCH program but were unable to attend. Individuals who have previously requested not to be contacted or have their records reviewed for potential participation in future research studies will not be contacted. Recruitment will be limited to Australian residents for this pilot study. Power analysis [30] suggests that 107 older adults will need to complete pre and post testing, given an expected effect size from meta-analysis of memory training [8] in older adults of Hedge's $g=$ 0.243 , with power $=0.80$, and alpha $=0.05$. Allowing for $70 \%$ retention of those who begin OPTIMiSE (based on the Wicking Dementia Research and Education Centre's Preventing and Understanding Dementia Massive Open Online Courses, MOOCs [31]), 153 participants will be recruited to the pilot study. Inclusion and exclusion criteria (see Table 1) were developed with the aim of recruiting a cohort of older Australians who self-perceive cognitive changes compared with 10 years ago, but who do not have a history of neurological or psychological conditions that are likely to have adversely impacted their cognition. All inclusion and exclusion criteria will be assessed based on participant self-report.

\section{Intervention}

The OPTIMiSE intervention is a free online 6module course, of approximately 2 hours content per module. Modules will be released weekly over 6 weeks and the program can be completed any time within a set 8 -week period. This will be followed by a single booster module of approximately 2 hours content delivered at 3-months post intervention, which can be accessed any time within a set 2-week period. The content of OPTIMiSE focuses on psychoeducation regarding models of memory, reasons for forgetting, and practical strategies that are effective for everyday memory difficulties.

Development of OPTIMiSE's content has been undertaken in consultation with a stakeholder advisory committee, involving individuals from the target recruitment group, and experts in health service delivery for older adults. A total of six one-hour meetings were held with the advisory committee throughout program development, in order to ensure that the pro-

Table 1

Inclusion and exclusion criteria

\begin{tabular}{ll}
\hline Inclusion Criteria & $\geq 60$ years of age \\
- Reporting subjective cognitive decline (respond yes to the question: "Do you think \\
your memory or thinking is worse than it was 10 years ago?") \\
- Sufficiently fluent in reading and typing English to access, read, and comprehend the course \\
material and participate in text-based online discussions \\
- Able to complete OPTIMiSE during the set 8-week period \\
- Able to complete the online evaluation questionnaires without assistance \\
- Diagnosis of dementia \\
- Diagnosis of a current psychiatric disorder likely to impact cognition \\
(e.g., psychotic illness or severe depression) \\
- History of any neurological condition likely to impact cognition or study participation \\
(e.g., stroke, cerebral palsy, epilepsy, multiple sclerosis, Parkinson's disease, \\
moderate or severe traumatic brain injury) \\
- Current alcohol or drug dependency
\end{tabular}


gram content meets the needs of older adults, that the program is easy to navigate and use, and that the outcome measures are appropriate. Development of OPTIMiSE proceeded in an iterative fashion with modifications to content and approach as per the advisory committee's suggestions. For example, one committee member suggested that it would be useful to include information on the relationship between sleep, memory, and ageing. After discussion, information on this topic was developed as part of Module 5. Some of the other advisory committee suggestions that were incorporated included considering the pacing of video content, being clear about the purpose of quizzes (to reinforce learning rather than to test knowledge) and providing clear instructions regarding navigation through the MOOC. The stakeholder advisory committee will also be consulted to discuss interpretation of the results and the next steps following completion of the pilot evaluation. A content advisory team was also created, consisting of clinical and academic experts in mental and cognitive health in older adults. The content advisory team contributed to the development of program content and methods of implementing content suitable for online delivery.

All core content will be delivered through the University of Tasmania Wicking Dementia Centre MO $\mathrm{OC}$ platform. This platform enables access via a range of devices including desktop computers, laptops, tablets, and smartphones. The learning platform has been designed to maximize accessibility for non-traditional adult learners, including those with low levels of previous education and/or computer and internet skills. The platform has been used to deliver the Wicking Centre's Understanding Dementia and Preventing Dementia MOOCs, which each attracted over 25,000 enrolments in their most recent iterations, and the Menzies Institute for Medical Research Understanding Multiple Sclerosis MOOC. The average age of Preventing Dementia MOOC participants is 50 and the probability of completing the course increases with age, with participants reporting using what they have learned to make lifestyle changes to reduce dementia risk [31]. The Understanding Dementia MOOC significantly builds dementia knowledge regardless of educational background and previous dementia experience [33]. Both courses have exceptionally high completion rates and high levels of participant satisfaction $[31,33]$. Use of this existing, accessible, and effective learning platform reduces the resources required for this pilot study. The dedicated platform development and support team provide the necessary expertise to successfully implement and deliver the OPTIMiSE program. This partnership is expected to enhance the feasibility of upscaling the OPTIMiSE program to a larger cohort in the future.

OPTIMiSE contains a series of core modules designed to be taken by all participants, plus optional content, enabling participants to personalize the program to their own interests and goals. Core program content has been modified from key components of successful memory strategy interventions such as the face-to-face LaTCH program [18]. The design of the intervention was informed by the refined Theoretical Domains Framework (TDF) [32]. The TDF synthesized 33 theories and 128 key theoretical constructs related to behavior change into a single framework to inform intervention design and guide implementation. The refined TDF reflects the outcomes of content validity testing, resulting in a framework of fourteen domains with high relevance to the OPTIMISE intervention (i.e., Knowledge, Skills, Social/Professional Role and Identity, Beliefs about Capabilities, Optimism, Beliefs about Consequences, Reinforcement, Intentions, Goals, Memory, Attention and Decision Processes, Environmental Context and Resources, Social Influences, Emotions, and Behavioral Regulation).

All participants will be directed to complete the six core modules, with topics as detailed in Table 2. OPTIMiSE covers simple models of memory to enhance understanding of reasons for forgetting, which are based on those presented in the LaTCH program and relates to the TDF domain of Knowledge. There is a component on memory across the lifespan-including changes with normal ageing compared to dementia (TDF Belief about Capabilities; Optimism). Participants will also be taught about evidence-based memory strategies including spaced retrieval, association, implementation intentions, PQ RST, as well as external aids (TDF domains of Skills; Goals). They will learn about the importance of attention, reducing the amount to remember, creating SMART goals, considering the learning environment, managing well-being including sleep, and remaining calm. Many, but not all, of these strategies are also covered in the LaTCH program. Strategies will be introduced using videos, text, and diagrams as appropriate. Participants will be provided with examples of how to use core strategies, and then given examples to try themselves within the module (TDF Skills). They will also have the opportunity to practice strategies in their everyday life for homework (TDF Skills). Core strategies are often repeated across multiple modules, as they are often relevant for more than one memory 
Table 2

Overview of OPTIMiSE module content

\begin{tabular}{ll}
\hline Module & Topics \\
\hline 1 & - The processes of memory \\
& - Strategies for learning new information \\
- The stages of memory \\
- The neuroscience of memory (optional) \\
- Strategies for remembering names \\
- Memory across the lifespan \\
- Strategies for remembering things we want or need to do \\
- Well-being in later life \\
- Strategies for how not to lose things and how to find things once lost \\
- Sleep in later life \\
- Strategies for remembering words and conversations; passwords and PINS. \\
- Strategies for finding your way (optional) \\
- Review \\
- Strategies for memory in everyday life \\
- Reflection and feedback \\
- Review focused on use of strategies in everyday life during the 3-month break
\end{tabular}

issue. A problem-solving approach will be suggested to manage any everyday memory difficulties, which involves understanding why the difficulty is happening and then applying the best strategy from those they have learned.

In terms of creating a personalized experience, this will be achieved by having optional extras for participants to access if they wish (for example additional external resources such as videos, articles, or help sheets), having some topics identified as optional extras, including the neuroscience of memory and strategies for navigation, and by providing participants with Customised Activities. At the beginning of OPTIMiSE, participants will be asked to identify their most important memory difficulty to work on from the following options: remembering names, losing items, remembering to do things (prospective memory), remembering the word you want, or learning and recalling new information (TDF domain Goals). At the end of each module, participants will be provided with a Customised Activity-homework specific to their memory priority. Participants will be asked to complete the Customised Activity relevant to their nominated priority but will also be able to complete any of the other Customised Activities that they choose.

Participants will be provided optional homework tasks after each module, incorporating both the core content and tasks focused on their individual memory priorities. These tasks will involve either reflecting on content presented in the Module and how it relates to the individual or implementing some of the strategies presented in the Module in their life. Participants will be asked to write their responses to these tasks on the discussion board.
OPTIMiSE will present information using a conversational framework, and in a variety of formats, including text, static and interactive diagrams and photographs, summaries and transcripts, animations of characters experiencing memory problems and employing strategies, videos of experts speaking about topics, and moderated online discussion boards. It will be interactive, with quizzes to reinforce learning, online practice of memory strategies, and exercises to practice in everyday life. Participants will be encouraged to take part in the moderated online discussion boards every week, to interact with other participants, observe and model strategy use with peers, and help foster a sense of community and peer support (TDF Social Identity; Beliefs about Capability; Social Influences). Discussion boards will be entirely text-based, and all boards will be accessible by all participants. Participants can elect to be identified by their first name and last initial, along with a small photograph of themselves, or they can choose to participate in discussions completely anonymously, with no name or photograph. Discussions will be moderated by study personnel, who will routinely check that posts adhere to stated guidelines to help ensure a welcoming and safe online environment. Staff may remove posts that do not adhere to stated guidelines or that are potentially harmful.

\section{Outcome measures}

The primary outcomes of this pilot study are feasibility, acceptability, and efficacy. Intervention feasibility will be assessed on a priori determined criteria recommended for use in feasibility studies: recruitment, acceptability, attrition, and data collection [34]. 
The primary measure of acceptability is whether participants would recommend OPTIMiSE to others. This question is asked as part of a post-course evaluation questionnaire (Supplementary Material 1), completed after finishing the entire OPTIMiSE program. This questionnaire provides additional information to further understand acceptability and feasibility of the program by asking about the online learning experience, the experience of online discussion board participation, and applicability of the course content to everyday life. Participants will also complete a brief evaluation questionnaire (Supplementary Material 2) following each module, assessing which parts of the module participants found most useful, and allowing identification of areas for potential program improvement. Further understanding of study feasibility will be gained through examination of which activities participants complete within each module, and amount of time spent on specific activities.

Intervention efficacy will be assessed using the following outcome measures: Memory Strategy Knowledge [35]; the Multifactorial Memory Questionnaire (MMQ), comprising three scales: MMQ-Satisfaction, MMQ-Ability, MMQ-Strategy [36]; Knowledge of Memory Ageing Questionnaire [37]; and questions relating to participants' personal memory goals (Supplementary Material 3). Note that Memory Strategy Knowledge and the three MMQ scales have previously been used to demonstrate efficacy of the LaTCH program [18]. All the efficacy measures will be administered at three timepoints: 1) prior to commencement of OPTIMiSE; 2) upon course completion; and 3) at the booster session 3-months post intervention, in order to assess maintenance of effect. To further understand the intervention's efficacy, at the booster session, participants will also be asked to provide information regarding how they were able to apply the knowledge and the strategies taught in their everyday life, as well as which strategies they use the most and in which situations, and which strategies they have not found useful.

The secondary aim of the pilot is to assess the sense of community created among participants, participants' feelings of control and self-efficacy regarding their memory ability, and psychological well-being, specifically the negative emotional states of depression, anxiety, and stress. Outcome measures used to assess these secondary aims are the Memory Controllability Inventory [38], a novel Memory Self-efficacy Questionnaire (Supplementary Material 4), a modified version of the Brief Sense of Community
Scale [39], and the 21-item version of the Depression Anxiety Stress Scale (DASS-21) [40]. Memory controllability, self-efficacy, and psychological wellbeing will be measured prior to the course, and all four measures will be administered following completion of the course, and at the 3-month booster session, to assess dimensions of needs fulfillment, group membership, influence, and shared emotional connection, in the context of an online community.

\section{Statistical analysis}

Feasibility will be measured by recruitment rates (number of participants giving informed consent from number expressing interest; consideration of number of organizations contacted; time frame for recruitment), acceptability (percentage of agree or strongly agree that they would recommend OPTIMiSE to others; suggestions for improvements), attrition (percentage of recruited participants completing OPTIMiSE), and data collection (amount and nature of missing data). Efficacy will be determined by a series of repeated measures $t$-tests, with alpha adjusted according to the Holm method, on pre- and post-performance on the outcome measures (personal memory goals, knowledge regarding memory and ageing, strategy knowledge, strategy use, memory ability, memory satisfaction, and psychological wellbeing). A descriptive summary will be provided of how the effect sizes on relevant outcome measures in OPTIMiSE compare to LaTCH. Effect sizes will be provided as Cohen's $d$, where $d=0.2$ is considered small, 0.5 is considered medium, and 0.8 is considered a 'large' effect size [41]. Thematic analysis, following the most significant change technique [42] will be used to analyze participants' responses to the question "What, if any, significant changes have you noticed in your life following the completion of the course?". Resulting themes will be compared to those previously reported following LaTCH memory groups [20, 21].

Pearson correlations will be conducted to identify associations between self-reported sense of community and levels of engagement on the OPTIMiSE discussion boards. Multiple regression analyses will be conducted to determine whether self-efficacy measured post-performance is predicted by demographic variables (age, gender, education) along with memory controllability and sense of community. Effect maintenance will be determined by repeated measures $t$-tests (again, using the Holm method for multiple 
comparisons) comparing outcome measures at the booster session versus immediately after the course.

\section{CONCLUSION}

Although we know that face-to-face memory interventions for older adults are effective, widespread translation to practice has been hampered by issues with sustainability of service delivery as well as accessibility. These issues have been intensified by the COVID-19 pandemic where non-urgent face-toface services are often being eliminated or avoided, particularly for older adults, who are at particularly high risk of significant negative consequences of this disease [23]. Online interventions provide a feasible solution to these issues [16]. An online memory strategy program will be developed in an iterative process through consultation with a stakeholder advisory committee. This program will then be executed, and we will evaluate its acceptability to older adults with cognitive concerns, the feasibility of running it on a larger scale, and whether it achieves similar outcomes to face-to-face groups. Comparison of effect sizes observed in the online pilot study to those reported in face-to-face groups [18] will enable us to determine whether an online format is as effective as face-to-face, across several different outcomes. That is, can an online format lead to moderate to large effect size changes in memory strategy knowledge and use, memory ability, and satisfaction with memory. Roadblocks or barriers along the way will inform future development. OPTIMiSE is the initial step in what could be an important program enabling access to an evidence-based and effective memory intervention, that improves knowledge, selfefficacy, and everyday memory performance, to older adults worldwide. These types of interventions have the potential to reduce memory-related anxiety and increase functional independence. The online learning platform used to deliver OPTIMiSE has already proven successful with programs increasing dementia knowledge and encouraging dementia risk reduction. Information from this proof-of-concept study will be used to modify OPTIMiSE before rolling it out to a larger audience, with further evaluation of this format for widespread translation of knowledge regarding effective memory strategies, and the facilitators and barriers of implementation. The ageing population will mean greater numbers of older adults living with cognitive concerns and an online accessible memory strategy program like OPTIMiSE has the potential to help older adults maintain independence for longer and reduce the growing burden on aged care services. This will be particularly useful during the current pandemic and in a post COVID-19 world.

\section{ACKNOWLEDGMENTS}

This project was funded by a La Trobe University Building Healthy Communities Research Funding Area Grant Ready grant awarded to KP.

We would like to acknowledge the members of our stakeholder advisory committee for their input into development of OPTIMiSE - Dr Jim Crosthwaite, Robyn Hartley, Ms Jenny Phillip, and Dr Anne Unkenstein.

\section{CONFLICT OF INTEREST}

The authors have no conflict of interest to report.

\section{SUPPLEMENTARY MATERIAL}

The supplementary material is available in the electronic version of this article: https://dx.doi.org/ 10.3233/ADR-200251.

\section{REFERENCES}

[1] Mewton L, Sachdev P, Anderson T, Sunderland M, Andrews G (2014) Demographic, clinical, and lifestyle correlates of subjective memory complaints in the Australian population. Am J Geriatr Psychiatry 22, 1222-1232.

[2] Slavin MJ, Brodaty H, Kochan NA, Trollor JN, Draper B, Sachdev PS (2010) Prevalence and predictors of "subjective cognitive complaints" in the Sydney memory and ageing study. Am J Geriat Psychiatry 18, 701-710.

[3] Petersen RC, Lopez O, Armstrong MJ, Getchius TSD, Ganguli M, Gloss D, Gronseth GS, Marson D, Pringsheim T, Day GS, Sager M, Stevens J, Rae-Grant A (2018) Practice guideline update summary: Mild cognitive impairment: Report of the Guideline Development, Dissemination, and Implementation Subcommittee of the American Academy of Neurology. Neurology 90, 126-135.

[4] Mitchell AJ, Beaumont H, Ferguson D, Yadegarfar M, Stubbs B (2014) Risk of dementia and mild cognitive impairment in older people with subjective memory complaints: Meta-analysis. Acta Psychiatr Scand 130, 439-451.

[5] Hurt CS, Burns A, Barrowclough C (2011) Perceptions of memory problems are more important in predicting distress in older adults with subjective memory complaints than coping strategies. Int Psychogeriatr 23, 1334-1343.

[6] Mol ME, van Boxtel MP, Willems D, Verhey FR, Jolles J (2009) Subjective forgetfulness is associated with lower quality of life in middle-aged and young-old individuals: A 9-year follow-up in older participants from the Maastricht Aging Study. Aging Ment Health 13, 699-705. 
[7] Hussenoeder FS, Conrad I, Roehr S, Fuchs A, Pentzek M, Bickel H, Moesch E, Weyerer S, Werle J, Wiese B, Mamone S, Brettschneider C, Heser K, Kleineidam L, Kaduszkiewicz H, Eisele M, Maier W, Wagner M, Scherer M, König H, Riedel-Heller SG (2020) Mild cognitive impairment and quality of life in the oldest old: A closer look. Qual Life Res 29, 1675-1683.

[8] Mewborn CM, Lindbergh CA, Miller LS (2017) Cognitive interventions for cognitively healthy, mildly impaired, and mixed samples of older adults: A systematic review and meta-analysis of randomized-controlled trials. Neuropsychol Rev 27, 403-439.

[9] Smart CM, Karr JE, Areshenkoff CN, Rabin LA, Hudon C, Gates N, Ali JI, Arenaza-Urquijo EM, Buckley RF, Chetelat G, Hampel H, Jessen F, Marchant NL, Sikkes SAM, Tales A, van der Flier WM, Wesselman L, the Subjective Cognitive Decline Initiative (SCD-I) Working (2017) Group Non-pharmacologic interventions for older adults with subjective cognitive decline: Systematic review, meta-analysis, and preliminary recommendations. Neuropsychol Rev 27, 245-257.

[10] Bhome R, Berry AJ, Huntley JD, Howard RJ (2018) Interventions for subjective cognitive decline: Systematic review and meta-analysis. BMJ Open 8, e021610.

[11] Chandler M, Parks A, Marsiske M, Rotblatt L, Smith G (2016) Everyday impact of cognitive interventions in mild cognitive impairment: A systematic review and metaanalysis. Neuropsychol Rev 26, 225-251.

[12] Hudes R, Rich JB, Troyer AK, Yusupov I, Vandermorris S (2019) The impact of memory-strategy training interventions on participant-reported outcomes in healthy older adults: A systematic review and meta-analysis. Psychol Aging 34, 587-597.

[13] van Heugten CM, Ponds RWHM, Kessels RPC (2016) Brain training: Hype or hope? Neuropsychol Rehabil 26, 639-644.

[14] McDaniel MA, Bugg JM (2012) Memory training interventions: What has been forgotten? J Appl Res Mem Cogn 1, 45-50.

[15] Barrios PG, Gonzalez RP, Hanna SM, Lunde AM, Fields JA, Locke DE, Smith GE (2016) Priority of treatment outcomes for caregivers and patients with mild cognitive impairment: Preliminary analyses. Neurol Ther 5, 183-192.

[16] Pike KE, Chong MS, Hume CH, Keech BJ, Konjarski M, Landolt KA, Leslie BE, Russo A, Thai C, Vilsten JS, Kinsella GJ (2018) Providing online memory interventions for older adults: A critical review and recommendations for development. Aust Psychol 53, 367-376.

[17] Lally P, Van Jaarsveld CH, Potts HW, Wardle J (2010) How are habits formed: Modelling habit formation in the real world. Eur J Soc Psychol 40, 998-1009.

[18] Kinsella GJ, Ames D, Storey E, Ong B, Pike KE, Saling MM, Clare L, Mullaly E, Rand E (2016) Strategies for improving memory: A randomized trial of memory groups for older people, including those with mild cognitive impairment. J Alzheimers Dis 49, 31-43.

[19] Kinsella GJ, Mullaly E, Rand E, Ong B, Burton C, Price S, Phillips M, Storey E (2009) Early intervention for mild cognitive impairment: A randomised controlled trial. J Neurol Neurosurg Psychiatry 80, 730-736.

[20] Matthews ML, Wells Y, Pike KE, Kinsella GJ (2020) Longterm effects of a memory group intervention reported by older adults. Neuropsychol Rehabil 30, 1044-1058.

[21] Kinsella GJ, Mullaly E, Rand E, Pike KE, Reilly A, Cavuoto MG (2020) Bridging the gap between clinical trials and community care: Translating a memory group for older people with mild cognitive impairment into a communitybased organisation. Australas J Ageing 39, e168-e177.

[22] Vranic A, Spanic AM, Carretti B, Borella E (2013). The efficacy of a multifactorial memory training in older adults living in residential care settings. Int Psychogeriatr 25, 1885-1897.

[23] Fisk M, Livingstone A, Pit SW (2020) Telehealth in the context of COVID-19: Changing perspectives in Australia, the United Kingdom, and the United States. J Med Internet Res 22, e19264.

[24] Australian Bureau of Statistics (2018) Household use of information technology, Australia, 2016-17 (Cat. No. 8146.0), ABS, Canberra.

[25] LaMonica HM, English A, Hickie IB, Ip J, Ireland C, West S, Shaw T, Mowszowski L, Glozier N, Duffy S, Gibson AA, Naismith SL (2017) Examining internet and ehealth practices and preferences: Survey study of Australian older adults with subjective memory complaints, mild cognitive impairment, or dementia. J Med Internet Res 19, e358.

[26] Evans SR (2010) Clinical trial structures. J Exp Stroke Transl Med 3, 8-18.

[27] von Elm E, Altman DG, Egger M, Pocock SJ, Gotzsche PC, Vandenbroucke JP, STROBE Initiative (2007) The Strengthening the Reporting of Observational Studies in Epidemiology (STROBE) statement: Guidelines for reporting observational studies. Lancet 370, 1453-1457.

[28] Eldridge SM, Chan CL, Campbell MJ, Bond CM, Hopewell S, Thabane L, Lancaster GA (2016) CONSORT 2010 statement: Extension to randomised pilot and feasibility trials. BMJ 355, i5239.

[29] Lancaster GA, Thabane L (2019) Guidelines for reporting non-randomised pilot and feasibility studies. Pilot Feasibility Stud 5, 114.

[30] Faul F, Erdfelder E, Lang AG, Buchner A (2007) G*Power 3: A flexible statistical power analysis program for the social, behavioral, and biomedical sciences. Behav Res Methods 39, 175-191.

[31] Farrow M, Doherty K, McInerney F, Klekociuk S, Bindoff A, Vickers J (2018) Improving knowledge and practice through massive open online dementia education: The Understanding Dementia and Preventing Dementia MOOCs. Alzheimers Dement 14, 233.

[32] Cane J, O'Connor D, Michie S (2012) Validation of the theoretical domains framework for use in behaviour change and implementation research. Implement Sci 7, 37.

[33] Eccleston C, Doherty K, Bindoff A, Robinson A, Vickers J, McInerney F (2019) Building dementia knowledge globally through the Understanding Dementia Massive Open Online Course (MOOC). NPJ Sci Learn 4, 3.

[34] Moore CG, Carter RE, Nietert PJ, Stewart PW (2011) Recommendations for planning pilot studies in clinical and translational research. Clin Transl Sci 4, 332-337.

[35] Hutchens RL, Kinsella GJ, Ong B, Pike KE, Parsons S, Storey E, Ames D, Saling MM, Mullaly E, Rand E, Clare L (2012) Knowledge and use of memory strategies in amnestic mild cognitive impairment. Psychol Aging 27, 768-777.

[36] Troyer AK, Rich JB (2018) Multifactorial Memory Questionnaire: Professional manual. Toronto: Baycrest Centre for Geriatric Care. Retrieved from http://www.baycrest. org $/ \mathrm{mmq}$

[37] Cherry K, West R, Reese C, Santa Maria M, Yassuda M (2000) The knowledge of memory aging questionnaire. Educ Gerontol 26, 195-219. 
[38] Lachman ME, Bandura M, Weaver SL, Elliott E (1995) Assessing memory control beliefs: The memory controllability inventory. Aging $\operatorname{Cogn}$ 2, 67-84.

[39] Peterson NA, Speer PW, McMillan DW (2008) Validation of a brief sense of community scale: Confirmation of the principal theory of sense of community. J Community Psychol 36, 61-73.

[40] Lovibond SH, Lovibond, PF (1995) Manual for the Depression Anxiety Stress Scales (2nd. Ed.). Psychology Foundation, Sydney.
[41] Cohen J (1988) Statistical Power Analysis for the Behavioral Sciences. Lawrence Erlbaum Associates, Hillsdale, NJ.

[42] Dart J, Davies R (2003) A dialogical, story-based evaluation tool: The most significant change technique. Am J Eval 24, 137-155. 


\section{University Library}

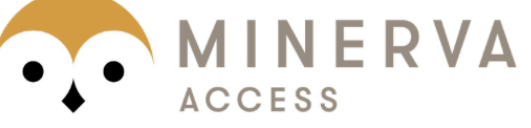

A gateway to Melbourne's research publications

Minerva Access is the Institutional Repository of The University of Melbourne

Author/s:

Pike, KE;Moller, C;Bryant, C;Farrow, M;Dao, DP;Ellis, KA

Title:

Online Personalised Training in Memory Strategies for Everyday (OPTIMiSE) Program for Older Adults with Cognitive Concerns: Pilot Study Protocol

Date:

2021-01-01

Citation:

Pike, K. E., Moller, C., Bryant, C., Farrow, M., Dao, D. P. \& Ellis, K. A. (2021). Online Personalised Training in Memory Strategies for Everyday (OPTIMiSE) Program for Older Adults with Cognitive Concerns: Pilot Study Protocol. JOURNAL OF ALZHEIMERS DISEASE REPORTS, 5 (1), pp.143-152. https://doi.org/10.3233/ADR-200251.

Persistent Link:

http://hdl.handle.net/11343/287162

License:

CC BY-NC 